\title{
INFANCIA, MORTALIDAD Y CAUSAS DE MUERTE EN ESPAÑA EN EL PRIMER TERCIO DEL SIGLO XX (1906-1932)
}

\author{
Alberto Sanz Gimeno
}

Universidad Complutense de Madrid

E-mail: asanzg@cps.ucm.es

\begin{abstract}
RESUMEN
Este trabajo aborda el estudio del declive de la mortalidad en la infancia ( 0 a 4 años) en la España del primer tercio del siglo XX, partiendo de la comparación de los niveles y estructura de la mortalidad por causas en dos fechas clave: 1906 y 1932. A partir del cálculo de la mortalidad infantil $\left(\mathrm{q}_{0}\right)$ y de la mortalidad juvenil $\left({ }_{4} \mathrm{q}_{1}\right)$ según los principales grupos de causas (enfermedades del aparato digestivo, respiratorio, infecto-epidémicas y del sistema nervioso), el trabajo aborda los cambios que se dieron en la mortalidad por edad en un momento clave del proceso de transición demográfica y de modernización social y económica. Así, se distinguen no sólo las causas de muerte relacionadas con el declive de la mortalidad, sino, también, algunos de los factores implicados en este descenso, realizando también un análisis de la distribución regional del fenómeno, que permite examinar algunas de las diferencias existentes entre diversas zonas del mapa de España.
\end{abstract}

\section{INTRODUCCIÓN ${ }^{1}$}

A nadie pasa desapercibida la importancia que ha tenido el descenso de la mortalidad en el proceso de transición y cambio de las pautas demográficas de

1 Este trabajo se ha desarrollado en el marco de investigación del Proyecto «La modernización demográfica y el declive de la mortalidad en la España urbana, 1860-1900» (Ministerio de Educación y Cultura - PB96/0637). El autor agradece a los profesores David-Sven Reher y Vicente Pérez Moreda su inestimable ayuda y el generoso préstamo de los datos de mortalidad 
la sociedad española. La relación de este fenómeno con el crecimiento de la población, el aumento de la esperanza de vida, la modificación de la fecundidad y el desarrollo social y económico, entre otras variables, otorga a su estudio un notable interés, especialmente en el caso de la mortalidad de los primeros años de vida o, más propiamente, de la infancia. No en vano, la mortalidad ocurrida entre el nacimiento y el quinto cumpleaños ( 0 a 4 años) suponía entre un tercio y la mitad de la mortalidad general durante el antiguo régimen demográfico. Por tanto, analizar la reducción de estas defunciones «infantiles» resulta esencial para entender buena parte de los cambios acontecidos en el proceso de modernización social y económica ocurrido a lo largo de los siglos XIX y XX.

En este estudio, el marco teórico de la transición demográfica constituye un referente obligado. La mortalidad y su caída ocupan un lugar central en esta teoría, siendo uno de los elementos básicos de los cambios demográficos y sociales experimentados a lo largo de dicho proceso de transición (Notestein, 1945: 39). Gracias a una reducción de la mortalidad se produciría un posterior ajuste del comportamiento reproductivo, ya que las familias verían sobrevivir, progresivamente, a más de sus hijos, adaptándose así a una nueva situación inserta en un proceso de cambios sociales y económicos. De este modo, la caída de la mortalidad abriría la puerta a un período de rápido crecimiento de la población y a un gradual ajuste de la fecundidad en una evolución desde altas a bajas tasas de natalidad y mortalidad.

En ese camino la reducción de la mortalidad está claramente ligada a la notable caída de la incidencia de las enfermedades infecciosas. La teoría de la transición epidemiológica, descrita por Omran en 1971, explica el paso desde un patrón epidemiológico caracterizado por el protagonismo de las enfermedades infecciosas a otro donde predominan las enfermedades no infecciosas, crónicas y degenerativas. El peso de uno u otro tipo de enfermedades establece, en buena medida, el grado de desarrollo y de mejoras alcanzado por una sociedad y constituye un referente clave para entender el proceso de modernización. En este sentido, esta teoría constituye otro referente teórico ${ }^{2}$ clave para el estudio de la mortalidad y, por tanto, para este trabajo.

Obras como las de Marcelino Pascua (1934a), Antonio Arbelo Curbelo (1962) y, más recientemente, Rosa Gómez Redondo (1992) han abordado el descenso de la mortalidad en España, centrándose principalmente en las defunciones de menores de un año a lo largo del siglo XX. Gracias a ellos se conocen el nivel y estructura de la mortalidad infantil durante buena parte del

por causas con los que se ha elaborado este escrito. Este agradecimiento se hace también extensivo a Begoña Gómez Garay, Fernando Pastor García-Quismondo y Guillermo Reher.

${ }^{2}$ Más recientemente, otras aportaciones teóricas han venido a completar algunos aspectos no contemplados en el marco de la teoría de la transición epidemiológica. Tal es el caso de la teoría de la transición sanitaria, que analiza el papel desempeñado en la caída de la mortalidad por los cambios sociales, culturales y de comportamiento, buscando la explicación a las transformaciones acontecidas en la mortalidad durante el proceso de modernización (Robles et al., 1996: 126). 
proceso de transición ${ }^{3}$. Sin embargo, aún existen importantes cuestiones por tratar en el conocimiento de este fenómeno. La falta de atención a la mortalidad ocurrida a partir del primer año de vida es uno de los rasgos comunes de los trabajos antes citados ${ }^{4}$. Tampoco hay demasiada información acerca de los cambios en la estructura de la mortalidad por causas, siendo muy limitado el análisis de la evolución de la mortalidad por edad y causa. Marcelino Pascua (1934a y 1934b) aborda esta cuestión, aunque sus estudios se basan esencialmente en datos para el conjunto nacional y sus resultados por intervalos de edad no permiten confeccionar agrupaciones homogéneas de la mortalidad por distintas enfermedades. Su único análisis provincial se basa en los datos del trienio de 1924 a 1926, lo que limita seriamente la observación de un cambio en el mapa epidemiológico español. Por otra parte, Antonio Arbelo (1962) recoge las estadísticas de defunciones por edad y causa durante la infancia y, aunque trabaja según las principales rúbricas de causa de muerte, sólo lo hace con datos nacionales en el decenio de 1941 a 1950.

Recientemente han salido a la luz otros trabajos (Ramiro Fariñas, 1998; Reher et al., 1997; Sanz Gimeno, 1999) que abordan la estructura de la mortalidad por edad y causa, examinando detalladamente el proceso de transición epidemiológica y la vinculación entre las causas de muerte y algunos de los principales factores determinantes. Sin embargo, estos estudios o bien se basan en datos locales o bien, pese a trabajar con datos provinciales, no incluyen las causas de muerte en su análisis (Dopico y Reher, 1998).

El objetivo de este trabajo es aportar un conocimiento más preciso acerca de la estructura de la mortalidad en la infancia por edad y causas, así como sus variaciones regionales, en el primer tercio del siglo XX. Para ello, y dada la abundancia de información que se precisa tratar, se han seleccionado dos fechas clave en el proceso de transición. Por un lado, el año 1906, en el inicio del siglo XX, en una etapa caracterizada por unos niveles altos de mortalidad y cierta inestabilidad en el proceso de reducción de este fenómeno. La otra fecha de referencia es el año 1932, que, pese a situarse en una etapa intermedia en el declive de la mortalidad, se encuentra en un momento de clara estabilidad en esta caída y muestra ya importantes cambios.

La elección de ambas fechas está relacionada con la disponibilidad de información y por la semejanza que existe entre este período y los abarcados en varios de los trabajos antes citados. Este hecho permite la realización de comparaciones con algunos de los resultados ya publicados. Además, hay que señalar que dentro del período seleccionado se producen dos de los más importan-

3 Aunque la mayor parte del período de declive de la mortalidad infantil y juvenil transcurre a lo largo del siglo XX, el inicio de la caída se produce en la segunda mitad del siglo XIX, siendo algo más temprano en el caso de la mortalidad juvenil (1 a 4 años) (Ramiro Fariñas y Sanz Gimeno, 1999: 66).

${ }^{4}$ Sólo Arbelo Curbelo (1962) realiza un análisis detallado de la mortalidad a partir del primer aniversario, aunque utiliza criterios de agrupación de edades diferentes a los usados habitualmente en el análisis demográfico. 
tes cambios en el declive de la mortalidad de los primeros años de vida. Así, a comienzos del siglo XX tiene lugar la transformación en la estructura por edad de la mortalidad, pasando la mortalidad infantil (menores de un año) a ser mayor que la juvenil (uno a cuatro años) (Ramiro Fariñas y Sanz Gimeno, 1999: 67). Por otro lado, a mediados de los años veinte, muy cerca de nuestra segunda fecha de referencia, la tradicional sobremortalidad urbana deja paso a una mayor mortalidad en los núcleos rurales (Pascua, 1934a: 53; Gómez Redondo, 1992: 74; Dopico y Reher, 1998: 77).

El primer tercio del siglo XX presenta, además, cambios sociales y económicos importantes, ligados al proceso de modernización de nuestro país. Así, junto a los progresos urbanísticos pueden destacarse la transformación en la valoración social de la figura del niño y un mayor nivel educativo de las madres, vinculado a la reducción de las tasas de analfabetismo femenino (Sanz Gimeno, 1999: 107-109). Asimismo, en el campo de la medicina las mejoras se producen básicamente en la atención higiénico-sanitaria.

Es en este marco de transformación y desarrollo, tanto en lo social como en lo económico, en el que se inserta este trabajo ${ }^{5}$, que parte del análisis del patrón epidemiológico de la mortalidad en los primeros años de vida para profundizar en algunos de los aspectos esenciales del proceso de transición demográfica y en los factores y determinantes implicados en el mismo.

\section{FUENTES Y DATOS}

Para este estudio se han usado los datos de las estadísticas del Movimiento Natural de la Población, siendo los principales problemas encontrados, por un lado, el del subregistro de fallecidos en las primeras veinticuatro horas de vida y la carencia de información en las defunciones por edad exacta, en concreto en 1906, que impide la elaboración del cociente de mortalidad juvenil $\left({ }_{4} \mathrm{q}_{1}\right)$ a partir de los difuntos y supervivientes en cada aniversario. Así, para el cálculo de la mortalidad infantil $\left(\mathrm{q}_{0}\right)$ en 1906 y 1932 se ha compensado la ausencia de datos relativos a los nacimientos y las defunciones de los muertos en el primer día de vida, partiendo de la dinámica de ajuste propuesta por Fausto Dopico y David Reher (1998: 18). Al mismo tiempo, se ha tenido en cuenta la distinta distribución de los nacidos del denominador en el mismo año y el año anterior, aplicando una ponderación de los nacimientos que tiene en cuenta el nivel de la mortalidad infantil en cada año (Ramiro Fariñas, 1998).

El cálculo de la mortalidad juvenil $\left(\mathrm{q}_{1}\right)$ se ha realizado teniendo en cuenta la disponibilidad de datos en cada fecha. Así, para 1932 se han recogido los nacimientos y defunciones edad a edad en los años anteriores y se han seguido

5 Buena parte de los resultados que aquí se presentan se enmarcan dentro de una línea de investigación, mucho más amplia, que partiendo de la información de una gran base de datos pretende lograr un conocimiento exhaustivo acerca de la mortalidad y los cambios acontecidos en la misma en el primer tercio del siglo XX. 
los procedimientos habituales de cálculo (Shryock y Siegel, 1976: 240; Sanz Gimeno, 1999: 33). En cambio, dada la falta de datos para 1906 y en fechas anteriores, se han utilizado los datos de nacimientos y defunciones de menores de un año y de uno a cuatro años para estimar ${ }_{4} \mathrm{q}_{1}$ de acuerdo al procedimiento que Fausto Dopico y David Reher utilizan en el caso de las tablas de vida de 1910-11 (Dopico y Reher, 1998: 18).

La estimación de la mortalidad por causa de muerte se ha realizado multiplicando cada cociente de mortalidad $\left(\mathrm{n}_{\mathrm{n}}\right)$ por los difuntos a una edad y causa determinada y dividiendo el resultado por el total de difuntos en dicha edad. De esta manera, el cociente de mortalidad por causa obtenido es un indicador del peso relativo de dicha causa en la probabilidad de morir para ese grupo de edad.

En cuanto a la información sobre las causas de muerte, existen algunas diferencias en las rúbricas existentes para ambas fechas, debido a las revisiones que periódicamente se han ido realizando sobre la nomenclatura de enfermedades $^{6}$. Así, en 1906, los datos de defunciones por edad se agrupan en torno a 40 rúbricas de causa de muerte, mientras que en 1932 ya hay 43 rúbricas. Estos cambios en la clasificación obligan a realizar una homologación entre las rúbricas existentes en las dos fechas, con el fin de hacer comparables los resultados de la mortalidad por causas en uno y otro año. Asimismo, se ha hecho una selección de aquellas causas de muerte de mayor interés para el estudio de la mortalidad en la infancia, elaborando grupos homogéneos de causas, pero manteniendo la heterogeneidad entre las agrupaciones que conforman la clasificación (Vallin, 1987: 82).

A la hora de realizar el análisis de causas de muerte y la correspondiente agrupación de enfermedades, se ha tenido en cuenta el carácter infeccioso o no infeccioso de cada una de las rúbricas y su relevancia en el descenso de la mortalidad en los primeros años de vida. Con estos criterios se ha confeccionado una clasificación de causas de muerte (tabla 1), que contiene también la propuesta de homologación para hacer comparables los resultados obtenidos en 1906 y 1932.

Las enfermedades del aparato digestivo se encuentran estrechamente relacionadas con el estado de desarrollo en materia de sanidad e higiene públicas y, también, con la atención y cuidado maternos, especialmente en lo que respecta a la alimentación del niño (Dopico, 1985: 369)ㄱ. La relación entre enfermedades del

${ }^{6}$ Esta periódica revisión de la Clasificación Internacional de Enfermedades constituye uno de los principales problemas en el uso de los datos del Movimiento Natural de la Población (Reher y Valero Lobo, 1995: 89).

La inclusión de la «cirrosis del hígado» en este grupo de enfermedades en 1906 se explica por la dificultad para separar dentro de esta rúbrica los procesos morbosos hepáticos, ligados al desarrollo de infecciones propias del hígado (hepatitis), de las enfermedades asociadas a estilos de vida y problemas tales como el alcoholismo. De hecho, en 1906 no se distingue el alcoholismo de las enfermedades propias del hígado, tal y como ocurre en 1932. Por otra parte, otros autores también consideran la inclusión de esta enfermedad dentro del grupo de enfermedades del aparato digestivo (Bertillon, 1899: 25; Barona y Barea, 1996: 69). Asimismo, conviene señalar que el peso de la mortalidad infantil y juvenil por la rúbrica de "cirrosis del hígado» es muy pequeño y no llega a un valor del 2 por 1.000 en ${ }_{5} \mathrm{q}_{0}$ para 1906. 


\section{TABLA 1}

Agrupación de causas de muerte para el análisis de la mortalidad de la infancia y propuesta de homologación para los años 1906 y 1932

\section{Aparato digestivo}

(25) Afecciones del estómago (menos cáncer)

(26) Diarrea y enteritis (mayores de 2 años)

(27) Diarrea y enteritis (menores de 2 años)

(28) Hernias, obstrucciones intestinales

(29) Cirrosis del hígado
(29) Diarrea y enteritis

(30) Apendicitis

(31) Enfermedades del hígado y de las vías biliares

(32) Otras enfermedades del aparato digestivo
(13) Tuberculosis pulmonar

(21) Bronquitis aguda

(22) Bronquitis crónica

(23) Neumonía

(24) Otras enfermedades del aparato respiratorio

\section{Aparato respiratorio}

(10) Tuberculosis del aparato respiratorio

(26) Bronquitis

(27) Neumonía

(28) Otras enfermedades del aparato respiratorio, excepto tuberculosis

\section{Enfermedades infecto-epidémicas}
(1) Fiebre tifoidea (tifo abdominal)
(2) Tifo exantemático
(3) Fiebre intermitente y caquexia palúdica
(4) Viruela
(5) Sarampión
(6) Escarlatina
(7) Coqueluche
(8) Difteria y crup
(9) Gripe
(10) Cólera asiático
(11) Cólera nostras
(12) Otras enfermedades epidémicas

(1) Fiebre tifoidea y paratifoidea

(2) Tifus exantemático

(3) Viruela

(4) Sarampión

(5) Escarlatina

(6) Coqueluche

(7) Difteria

(8) Gripe

(9) Peste

(13) Paludismo (Malaria)

(14) Otras enfermedades infecciosas y parasitarias

\section{Enfermedades del sistema nervioso}

(18) Meningitis simple

(19) Congestión, hemorragia y reblandecimiento cerebrales
(21) Ataxia locomotriz progresiva y parálisis general

(22) Hemorragia cerebral, embolia o trombosis cerebral

(23) Otras enfermedades del sistema nervioso y de los órganos de los sentidos

\section{Enfermedades perinatales y congénitas}

(35) Debilidad congénita y vicios de conformación
(38) Debilidad congénita, vicios de conformación congénitos, nacimiento prematuro

NotA: Entre paréntesis figuran los números correspondientes a cada una de las rúbricas que aparecen en el Movimiento Natural de la Población de 1906 y 1932. 
aparato respiratorio y del sistema nervioso con las medidas higiénicas y sanitarias, e incluso con el desarrollo de terapias médicas o el cuidado de los alimentos, resulta difícil de establecer en algunos casos y en su caída hay que tener en cuenta el papel de otros elementos, como una menor virulencia de la enfermedad, cierta mejora en las condiciones de vida que dificulten la transmisión de los microorganismos y una mayor resistencia del organismo frente a estas enfermedades, proporcionada fundamentalmente por una mejor nutrición (McKeown, 1978).

También se ha confeccionado un grupo de enfermedades, denominado «infecto-epidémicas», en el que se encuentran diversas infecciones y en cuya caída cabe considerar la influencia de diversos elementos, desde la nutrición hasta el desarrollo de los avances científico-médicos. Su comportamiento virulento y epidémico, su dispar etiología y el distinto peso que ejercen algunas de estas enfermedades han aconsejado su análisis como categoría específica. Asimismo, se ha dedicado un espacio a la mortalidad perinatal y congénita, dada su importancia en las defunciones del primer año de vida, si bien hay que considerar los problemas existentes en el diagnóstico y tratamiento de estas enfermedades, dado el desarrollo de los conocimientos de la Obstetricia en el período considerado por este trabajo.

También conviene señalar algunas cuestiones relacionadas con el uso de la información para dos fechas concretas. Así, por un lado, pueden ponerse objeciones a la utilización de años concretos como puntos de referencia para hacer comparaciones en la reducción y cambio estructural de la mortalidad. Sin embargo, no existen grandes variaciones con los niveles de mortalidad calculados para los años más cercanos, y allí donde ha sido posible establecer la comparación los resultados son muy similares a los obtenidos en otros estudios ${ }^{8}$.

${ }^{8}$ En el cuadro 1 se han plasmado algunos resultados de la mortalidad en la infancia para los años inmediatamente anteriores y posteriores a 1906 y 1932. Los cambios en el nivel de cada indicador no superan el 10 por 100 (cuando se cuenta con información para hacer dicha comprobación). Si se comparan los resultados de este trabajo con los de otros estudios, las diferencias en los valores de la mortalidad infantil y juvenil no superan, en ningún caso, el 5 por 100.

CUADRO 1

Valores de la mortalidad infantil y juvenil (\%o) en España en torno a 1906 y 1932

\begin{tabular}{|c|c|c|c|c|c|c|c|c|c|c|}
\hline & 1904 & 1905 & 1906 & 1907 & 1908 & 1930 & 1931 & 1932 & 1933 & 1934 \\
\hline 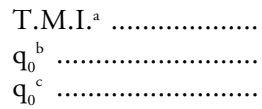 & . 172,9 & 161,3 & $\begin{array}{l}173,7 \\
183,6^{*} \\
180,2\end{array}$ & 158 & 159,9 & $\begin{array}{l}123,8 \\
124,0 \\
124,7\end{array}$ & 122,6 & 117,5 & 118,1 & 118,5 \\
\hline 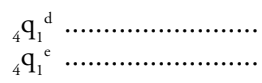 & & & $\begin{array}{l}179,6^{*} \\
172,6\end{array}$ & & & 89,0 & & 75,6 & & \\
\hline
\end{tabular}

NotAs:

a Tasa de mortalidad infantil (Gómez Redondo, 1992: 34).

b Mortalidad infantil $\left(\mathrm{q}_{0}\right)$ (Dopico y Reher, 1998: 32-36).

c Mortalidad infantil $\left(\mathrm{q}_{0}\right)$ elaborada para este trabajo.

d Mortalidad juvenil ( ${ }_{4} \mathrm{q}_{1}$ ) (Dopico y Reher, 1998: 32-36).

* El resultado correspondiente a 1906 es la media de $\mathrm{q}_{0}$ en 1900-01 y 1910-11.

e Mortalidad juvenil $\left({ }_{4} q_{1}\right)$ elaborada para este trabajo. 
También hay que tener presente que se trata de información para grandes agregados de población (provincias y capitales) y que, en ausencia de crisis específicas, las pequeñas variaciones en los niveles de mortalidad de un año para otro no tienen por qué suponer un cambio sustancial del comportamiento estructural del fenómeno.

Otro problema es el que atañe a la calidad diagnóstica, sobre todo en los primeros años del Movimiento Natural de la Población. Una prueba fehaciente de ello es el elevado peso que tiene la rúbrica de "otras enfermedades» en el año 1906. Así, en 1906 y para el conjunto nacional, el cociente de mortalidad infantil $\left(\mathrm{q}_{0}\right)$ por la categoría de "otras enfermedades» supone entre un 13 y un 14 por 100 del total de la mortalidad infantil y juvenil. Si a esta rúbrica añadimos el peso de la mortalidad por «enfermedades desconocidas y mal definidas», en 1906 resulta imposible explicar entre un 17 y un 18 por 100 de la mortalidad infantil y juvenil por causas, mientras que en 1932 este porcentaje se ha reducido hasta el 2 y el 3 por 100 . La puesta en práctica de técnicas de estimación para intentar paliar el problema requiere la adopción de hipótesis, a menudo, arriesgadas, siendo bastante complicado adoptar criterios homogéneos y válidos cuando se pretende trabajar sobre variaciones geográficas y temporales de los datos (Vallin, 1987: 134-137).

\section{LA ESTRUCTURA DE LA MORTALIDAD INFANTIL Y JUVENIL EN ESPAÑA}

En las fechas tomadas para nuestro análisis, y con los datos del Movimiento Natural de la Población, nos encontramos que, en 1906, la mortalidad en los primeros años de vida, pese al descenso experimentado a finales del siglo XIX, sigue siendo bastante importante. La proporción de difuntos entre 0 y 4 años se sitúa en torno al 43 por 100 de la mortalidad general, siendo calificada como "excesiva» por diversos autores de la época (Revenga, 1904: 62; Ulecia Cardona, 1903: 164-165). La mortalidad de los menores de un año $\left(\mathrm{q}_{0}\right)$ se sitúa ligeramente por encima de la mortalidad juvenil $\left({ }_{4} \mathrm{q}_{1}\right)$ (tabla 2). Esta estructura de la mortalidad, con un peso importante de la mortalidad juvenil, corresponde aún a una fase inestable en el proceso de descenso de este fenómeno, mostrando, también, el retraso del declive de la mortalidad española frente a otros países del norte y oeste de Europa, donde los valores de la mortalidad juvenil $\left(\mathrm{q}_{4}\right)$ se sitúan por debajo de la mortalidad infantil con anterioridad al comienzo del siglo $\mathrm{XX}$.

Estamos trabajando, por tanto, con los datos de unos años ajenos a fluctuaciones importantes de la mortalidad. Por otra parte, en lo que respecta a los valores de los indicadores de mortalidad presentados, conviene advertir que las diferencias existentes en los valores de la tasa de mortalidad infantil y el $\mathrm{q}_{0}$ calculado para este trabajo se explican por los diferentes procedimientos de cálculo empleados para ambos indicadores y, sobre todo, por el uso de la ecuación compensadora del subregistro de fallecidos en las primeras veinticuatro horas de vida para la elaboración del $\mathrm{q}_{0}$ (Dopico y Reher, 1998: 18). 


\section{TABLA 2}

Mortalidad infantil y juvenil (\%o) en España en 1906 y 1932

\begin{tabular}{|c|c|c|c|c|}
\hline & $q_{0}^{*}$ & $q_{1}^{*}$ & ${ }_{5} q_{0}^{*}$ & $\left(q_{0} / q_{4}\right)$ \\
\hline 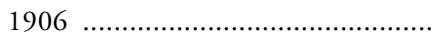 & 180,2 & 172,6 & 321,7 & 1,04 \\
\hline 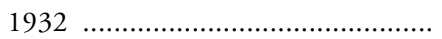 & 119,6 & 75,6 & 186,2 & 1,58 \\
\hline Porcentaje de reducción ................... & 34 & 56 & 42 & - \\
\hline
\end{tabular}

* $\mathrm{q}_{0}=$ mortalidad de menores de 1 año, ${ }_{4} \mathrm{q}_{1}=$ mortalidad de niños de 1 a 4 años $\mathrm{y}_{5} \mathrm{q}_{0}=$ mortalidad de niños entre 0 y 4 años cumplidos.

Elaboración propia a partir de los datos del $M N P$.

Casi treinta años después, en 1932, las defunciones de los primeros cinco años de vida representan el 31 por 100 de la mortalidad general. Esta proporción es el resultado de una importante reducción de la mortalidad en la infancia $\left({ }_{5} \mathrm{q}_{0}\right)$ desde 1906, que cae en algo más del 40 por 100 , dándose los principales avances en el caso de las defunciones de 1 a 4 años $\left({ }_{4} q_{1}\right)$ (tabla 2). Así, en 1932 el patrón de mortalidad infantil y juvenil es el propio de las poblaciones en plena transición demográfica, concentrándose la mayor parte de los fallecimientos en el primer año de vida. La fuerte caída de la mortalidad entre 1 y 4 años indica claramente la importancia de la reducción de las causas de origen exógeno, principales responsables de los fallecimientos ocurridos una vez cumplido el primer aniversario.

El análisis de estructura por causas de la mortalidad permite examinar con mayor detalle el proceso e identificar la naturaleza de los factores implicados en la caída de la mortalidad. Con este fin, y usando los datos del Movimiento Natural de la Población en 1906 y 1932, se han elaborado la tabla 3 y el gráfico 1 , que contienen diversos indicadores acerca de la estructura y evolución de la mortalidad por causas. La tabla 3 muestra los valores de los cocientes de mortalidad $\left(\mathrm{q}_{0},{ }_{4} \mathrm{q}_{1}\right.$ y $\left.{ }_{5} \mathrm{q}_{0}\right)$ para 1906 y 1932 en los principales grupos de causas de defunción en la infancia. En el gráfico 1 se han representado los porcentajes de caída de la mortalidad atribuibles a cada uno de los grupos de causas según la edad de los fallecidos. Respecto a este gráfico, hay que tener en cuenta la influencia que tienen el grupo de "otras enfermedades" y las «desconocidas y mal definidas» a la hora de restar capacidad explicativa a las causas consideradas en el descenso de la mortalidad.

Como puede apreciarse en la tabla 3, donde aparecen los niveles de mortalidad por edad, en ambas fechas la mortalidad por enfermedades del aparato digestivo, fundamentalmente diarrea y enteritis, supera a la relacionada con otro tipo de causas. Le siguen a cierta distinta las afecciones del aparato respiratorio, tales como bronquitis y neumonías, principalmente. Esta mayor incidencia de las enfermedades del aparato digestivo, unida a su clara etiología, 
pone de relieve la estrecha relación entre la mortalidad infantil y juvenil y los problemas de higiene y alimentación existentes en la España del primer tercio del siglo XX, tal y como ya puso de manifiesto Marcelino Pascua (1934a: 34). En 1906, dichos problemas eran especialmente importantes tanto antes como después del primer año de vida, mientras que ya en 1932 se hace bien patente la mayor reducción de la mortalidad juvenil $\left(\mathrm{q}_{1}\right)$ por este grupo de causas. $\mathrm{Si}$ examinamos el porcentaje de contribución de cada grupo de causas a la reducción de la mortalidad (gráfico 1) se observa que la reducción de la mortalidad por enfermedades del aparato digestivo contribuye, más que ninguna otra, a explicar la caída de la mortalidad en la infancia entre 1906 y 1932 , especialmente en el grupo de edad de 1 a 4 años.

\section{TABLA 3}
Mortalidad infantil y juvenil (\%o) según grupos de causa de muerte en España en 1906 y 1932

\begin{tabular}{cccc}
\hline \multicolumn{1}{c}{1906} & & \\
\hline Grupos de causa & $q_{0}{ }^{*}$ & ${ }_{4} q_{1}{ }^{*}$ & ${ }_{5} q_{0}{ }^{*}$ \\
\hline Enfermedades aparato digestivo .............. & 54,2 & 53,8 & 98,4 \\
Enfermedades aparato respiratorio ........... & 35,5 & 31,1 & 60,8 \\
Enfermedades infecto-epidémicas ........... & 13,2 & 28,9 & 38,1 \\
Enfermedades sistema nervioso ............... & 14,4 & 16,0 & 27,7 \\
Enfermedades perinatales y congénitas ...... & 26,6 & 1,6 & 26,3 \\
& & 172,6 & 321,7 \\
\hline
\end{tabular}

1932

\begin{tabular}{|c|c|c|c|}
\hline Grupos de causa & $q_{0}^{*}$ & ${ }_{4} q_{1}^{*}$ & ${ }_{5} q_{0}^{*}$ \\
\hline Enfermedades aparato digestivo .................... & 41,2 & 23,3 & 61,6 \\
\hline Enfermedades aparato respiratorio ............. & 27,7 & 22,2 & 47,3 \\
\hline Enfermedades infecto-epidémicas .............. & 6,0 & 10,2 & 15,2 \\
\hline Enfermedades sistema nervioso ................... & 9,4 & 8,6 & 17,0 \\
\hline Enfermedades perinatales y congénitas ...... & 26,2 & 0,5 & 26,2 \\
\hline 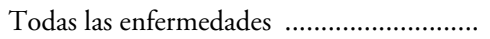 & 119,6 & 75,6 & 186,2 \\
\hline
\end{tabular}

* $\mathrm{q}_{0}=$ mortalidad de menores de 1 año, ${ }_{4} \mathrm{q}_{1}=$ mortalidad de niños de 1 a 4 años $y_{5} \mathrm{q}_{0}=$ mortalidad de niños entre 0 y 4 años cumplidos.

Elaboración propia a partir de datos del $M N P$.

${ }^{9}$ Las diferencias con otros trabajos y poblaciones, donde destaca la reducción de la mortalidad por enfermedades transmitidas a través del aire (McKeown, 1978: 87), puede explicarse por las diferencias en las edades empleadas en el estudio y los distintos criterios de clasificación utilizados. 
En la tabla 3 observamos que la incidencia de las enfermedades del aparato digestivo es especialmente importante en la mortalidad de los menores de un año. Este resultado es similar al obtenido en otros trabajos donde se ha destacado una mayor mortalidad por enfermedades del aparato digestivo (esencialmente diarrea y enteritis) entre el tercer y onceavo mes de edad y, también, durante el primer aniversario (Sanz Gimeno y Ramiro Fariñas, 2000). En esta etapa de la niñez cabe destacar como principales factores responsables de la mortalidad por este grupo de causas la introducción de una alimentación suplementaria a la lactancia, el destete y la dentición. Estos factores son de una importancia crucial de cara a las afecciones del aparato digestivo, sobre todo si se tiene en cuenta la precariedad de los hábitos y normas de higiene y la falta de cuidados adecuados por parte de las madres, que a menudo incurrían en prácticas erróneas en el tratamiento de las dolencias y problemas intestinales del niño (Bernabeu Mestre, 1994: 70-71; Perdiguero Gil, 1993: 74).

\section{GRÁFICO 1}

Contribución porcentual de los principales grupos de causas de muerte a la reducción de la mortalidad infantil y juvenil española entre 1906 y 1932

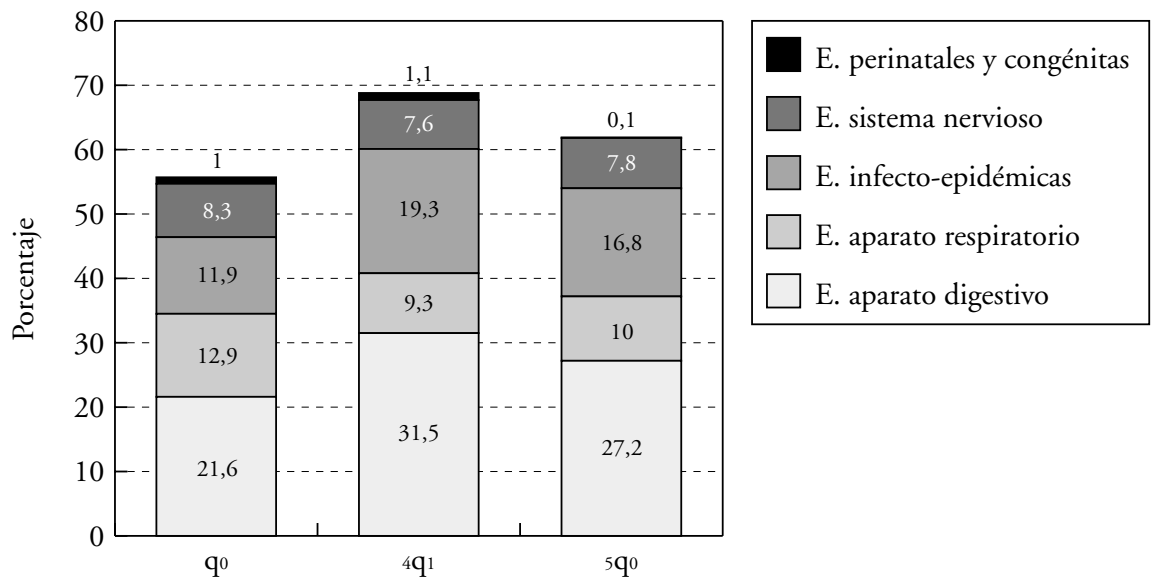

NotA: En el interior de cada segmento figura la contribución porcentual para cada cociente de mortalidad $\left(\mathrm{n}_{\mathrm{x}}\right)$ y causa de muerte.

Elaboración propia a partir de datos del $M N P$.

En su obra sobre La mortalidad infantil en España, Marcelino Pascua $(1934 a)$ dedica una especial atención a la evolución de la mortalidad por diarrea y enteritis en los menores de uno y dos años de edad, ya que descubre que la tendencia de la mortalidad por esta causa, lejos de descender, se man- 
tiene estable e incluso aumenta en determinados años (Pascua, 1934a: 34). Otros resultados procedentes de investigaciones más recientes también muestran la estabilidad de este grupo de causas durante buena parte de la primera mitad del siglo Xx. Dicha estabilidad se da en la mortalidad de los menores de un año, ya que la mortalidad juvenil experimenta una clara reducción (Ramiro Fariñas, 1998; Sanz Gimeno, 1999: 99; Sanz Gimeno y Ramiro Fariñas, 2000).

Por tanto, tal y como señala Pascua (1934a: 34), estamos ante una tendencia que alerta claramente sobre la gravedad que para la España de principios del siglo XX supone la falta de desarrollo en el área de las infraestructuras sanitarias y de la higiene, tanto pública como privada. A la luz de estos resultados, cabe preguntarse si el proceso de modernización tuvo realmente efectos sobre la mortalidad por este grupo de causas en el primer tercio de siglo y si se produjo un cambio de tendencia que pueda interpretarse como un logro de los avances conseguidos en dicho proceso de modernización. Los resultados de nuestro análisis sobre los datos del Movimiento Natural de la Población confirman que sí hubo, efectivamente, cierto proceso de caída en la probabilidad de morir por enfermedades del aparato digestivo entre 1906 y 1932 en el conjunto del país, lo que supone cierta mejora en materia de higiene y sanidad públicas entre estas fechas. Examinando los niveles del indicador (tabla 3) se observa cómo en los menores de un año la mortalidad por este grupo de causas se reduce en un 24 por 100, y la de los difuntos de 1 a 4 años lo hace en un 57 por 100. El contraste con los resultados ofrecidos por Pascua y con los que se desprenden de la investigación sobre la España interior tiene, no obstante, su explicación. En primer lugar, hay que destacar que el análisis de Pascua sólo llega hasta el año 1930, lo que constituye una primera diferencia en cuanto a las fechas tomadas como referencia. Así, si se toman los mismos datos y se calculan los mismos indicadores hasta 1932 es posible medir una reducción de la mortalidad infantil $\left(\mathrm{q}_{0}\right)$ por diarrea y enteritis del 26 por 100. En el caso de los menores de dos años la reducción es del 24 por 100 . De este modo, los resultados aquí presentados se confirman con los datos y cálculos propuestos por el propio Pascua.

No obstante, la reducción de la mortalidad infantil por enfermedades del aparato digestivo en el conjunto nacional contrasta con la estabilidad e incluso aumento de dicha mortalidad en las series locales del interior peninsular (muestra rural). En dichas series la mortalidad infantil por este grupo de causas se mantiene prácticamente estable hasta después de la Guerra Civil (Sanz Gimeno y Ramiro Fariñas, 2000). A la hora de explicar estas diferencias hay que tener en cuenta el carácter rural de las mencionadas series locales, mientras que los datos del conjunto nacional cuentan con un peso nada desdeñable de la población que vive en las capitales de provincia. Esta disparidad es importante, sobre todo si se tiene en cuenta que desde mediados de los años veinte las ciudades comienzan a experimentar los beneficios de las inversiones realizadas en infraestructuras de sanidad e higiene públicas, como redes de agua pota- 
ble y alcantarillado, mostrando una mayor reducción de la mortalidad que se traduce en la desaparición de la sobremortalidad urbana, que caracteriza las etapas anteriores e iniciales de la transición (Pascua, 1934a: 53; Arbelo, 1962; Gómez Redondo, 1992: 74; Reher, 1996: 175).

Así, la rapidez e intensidad del cambio en los factores que determinan la mortalidad se hace especialmente evidente en el caso de las poblaciones urbanas. Los datos del Movimiento Natural de la Población muestran que, entre 1906 y 1932, la caída de la mortalidad infantil urbana por enfermedades del aparato digestivo dobla a la del resto provincial (aproximación al mundo rural). Así, la mortalidad infantil $\left(\mathrm{q}_{0}\right)$ por enfermedades digestivas se reduce en un 41 por 100 para el conjunto nacional urbano, mientras que en el resto de poblaciones la caída es del 20 por 100 . Conviene tener en cuenta, además, que en ese resto provincial existen grandes entidades de población, que también se vieron beneficiadas por las mejoras higiénico-sanitarias y que contribuyen a explicar por qué cae la mortalidad por enfermedades del aparato digestivo, mientras que el mundo rural experimenta un mayor retraso en este sentido. Con todos estos resultados cabe concluir, por tanto, la existencia de una mejora en los factores que influyen en la mortalidad por enfermedades digestivas, siendo los avances más acusados a partir del primer aniversario y en las entidades de población de cierto tamaño (capitales).

Entre 1906 y 1932 también se produce una reducción importante, aunque menor, en la mortalidad por enfermedades del aparato respiratorio. Este grupo pasa a convertirse, junto a las enfermedades del aparato digestivo, en uno de los principales componentes en la mortalidad de 1 a 4 años. Conviene tener en cuenta la importancia que elementos ligados a una mejora en los cuidados infantiles, en el nivel de vida y en el estatus nutritivo han tenido en el descenso de la mortalidad por enfermedades tales como las bronquitis y las neumonías. Contra estas infecciones no existen terapias médicas adecuadas hasta la llegada de los antibióticos y las sulfamidas después de los años cuarenta. Además, en este tipo de enfermedades, esencialmente de transmisión aérea, no resulta posible establecer medios de control sobre su propagación. De este modo, más que la higiene y sanidad públicas, la alimentación y los cuidados infantiles, junto a mejoras del entorno, cobran una especial significación a la hora de explicar la caída de la mortalidad por este grupo de causas.

El papel de las enfermedades infecto-epidémicas, así como los otros grupos de enfermedades, debe evaluarse en función de su incidencia según la edad de los fallecidos y del año que se esté tratando. Así, en 1906, el peso de las enfermedades infecto-epidémicas, claro resquicio del patrón epidemiológico de mortalidad en el antiguo régimen demográfico, es considerable (Pérez Moreda, 1985: 64). Los resultados señalan su incidencia especialmente elevada en el caso de las defunciones entre 1 y 4 años, con un valor de ${ }_{4} \mathrm{q}_{1}$ igual a 29 por 1.000 (tabla 3). Para esta fecha, la viruela tiene un peso pequeño sobre la mortalidad de la infancia (el valor de ${ }_{5} \mathrm{q}_{0}$ en esta enfermedad es de 3,8 por 1.000), dados los avances conseguidos en las campañas de vacunación emprendidas en años ante- 
riores $^{10}$. Sin embargo, el sarampión sigue teniendo un valor alto ${ }_{5} \mathrm{q}_{0}$ tiene un valor de 10,4 por 1.000), ejerciendo mayor presión sobre los niños de 1 a 4 años, dado que esta enfermedad necesita de efectivos sin un contagio previo para su contagio y extensión (Burnet y White, 1982: 29-31). Le siguen en importancia enfermedades como la difteria, que se encontraba en franco retroceso incluso antes de la extensión del suero antidiftérico a finales del siglo XIX (Rodríguez Ocaña, 1996: 163). Otras afecciones de menor consideración son la coqueluche o tos ferina y la gripe, cuyo diagnóstico ofrece a menudo ciertas dudas acerca de su peso efectivo sobre la mortalidad (Pascua, 1934a: 29; Echeverri Dávila, 1993: 4).

En 1932, el peso de las enfermedades infecto-epidémicas se ha reducido considerablemente tanto en la mortalidad infantil $\left(\mathrm{q}_{0}\right)$ como, y sobre todo, en la juvenil $\left({ }_{4} \mathrm{q}_{1}\right)$. Este cambio indica claramente la vinculación de este tipo de enfermedades a las condiciones de vida que caracterizaban las poblaciones del pasado y las primeras etapas de la transición. Su declive se convierte en un claro indicador de modernización social y económica. En su caída no cabe hablar en todos los casos de una eficaz intervención médica, dado que sólo en la viruela se ha demostrado una clara relación entre la desaparición de esta enfermedad y las campañas de vacunación masiva. Incluso la difteria comienza a caer antes del descubrimiento y utilización del suero antidiftérico (Sanz Gimeno y Ramiro Fariñas, 2000). Pero en el resto de enfermedades resulta más difícil establecer la naturaleza de los factores implicados en su descenso. Se ha hablado de un cambio en la relación huéspedparásito en el caso de enfermedades como la escarlatina (McKeown, 1978: 101), aunque no cabe decir lo mismo de otras afecciones tan importantes como el sarampión. En la reducción de esta y otras enfermedades hay que señalar de nuevo los beneficios de unos mayores y mejores cuidados maternos y, por supuesto, una mejora en las condiciones de vida, que incluyen la nutrición como uno de los determinantes clave. No hay que olvidar que a principios del siglo XX el hambre todavía se citaba como una de las principales causas de mortalidad (Pérez Moreda, 1985: 62). Con todo, tampoco puede olvidarse la estrecha interrelación del factor nutritivo con otros elementos, incluida la propia naturaleza de las enfermedades (Livi-Bacci, 1987: 59).

Las enfermedades del sistema nervioso, esencialmente meningitis, exhiben una importante reducción, también más acusada en ${ }_{4} \mathrm{q}_{1}$. Sin embargo, y pese a su importancia relativa, su contribución al descenso de la mortalidad (gráfico 1) es menor que en los grupos de causas vistos hasta el momento. Resulta difícil establecer una relación entre su descenso y la mejora de ciertos factores, dada la etiología variable de este tipo de enfermedades. Por otra parte, cabe considerar problemas diagnósticos, dado que se trata de una enfermedad de difícil definición en la época tratada, y su alto peso sobre la mortalidad infantil

${ }_{10}$ Aunque la inmunización contra la viruela se conocía desde finales del siglo XVIII, todavía en 1866 el 58 por 100 de los niños españoles no habían sido vacunados (Nadal, 1991: 112). En 1902 persisten las denuncias acerca de la falta de medidas administrativas para procurar la vacunación masiva de la población (Hauser, 1902: 48-49). 
y juvenil española frente a otros países europeos hace sospechar acerca de la confusión existente con otras enfermedades, tales como la fiebre tifoidea y la tuberculosis (Pascua, 1934b: 101-102).

Por último, y como señala Marcelino Pascua en los resultados de su trabajo (1934a: 30), cabe destacar que las enfermedades perinatales y congénitas, asociadas a la mortalidad cercana al nacimiento, no muestran descenso alguno, manteniendo su nivel estable a lo largo del período considerado. En esta evolución conviene tener en cuenta tanto la influencia de la calidad diagnóstica como el hecho de que se trata de procesos morbosos, principalmente de carácter endógeno, en los que resulta bastante difícil una intervención médico-sanitaria eficaz en el período considerado. Los avances en la Obstetricia habrían contribuido a mantener el nivel de estas enfermedades al propiciar la mejora en la identificación y el diagnóstico de las mismas.

Estos resultados nacionales no dejan dudas acerca de la composición y cambios del patrón epidemiológico general durante esta fase de la transición. Sin embargo, a la luz de lo que se sabe sobre las variaciones espaciales de la mortalidad infantil y juvenil, cabe esperar que tanto el perfil de la mortalidad por causas como su transformación hayan seguido distintas pautas de comportamiento en las diferentes áreas geográficas que define el mapa provincial. En el siguiente apartado se tratará esta cuestión, evaluando la importancia de cada causa de muerte a la hora de explicar la reducción de la mortalidad infantil y juvenil y su distribución geográfica.

\section{VARIACIONES ESPACIALES EN LA MORTALIDAD DE LA INFANCIA Y SUS CAUSAS}

Tal y como ya ha sido puesto de relieve en los trabajos de Marcelino Pascua (1934a), Rosa Gómez Redondo (1992) y Sanz Gimeno y Ramiro Fariñas (1999), entre otros, el patrón geográfico de la mortalidad infantil y juvenil española a principios del siglo XX (mapa 1) se caracteriza por la existencia de niveles altos en las zonas del interior (ambas Castillas y Extremadura) y el sur peninsular (Andalucía), frente a una baja mortalidad en el área de Galicia, la Cornisa Cantábrica, Cataluña y Baleares, dándose niveles intermedios en el Levante y algunas de las provincias de la mitad norte interior. En 1932 (mapa 2), la situación es similar aunque con algunas diferencias relacionadas con el proceso de aumento de las variaciones relativas, que acompaña la caída de la mortalidad infantil y juvenil ${ }^{11}$. No obstante, los niveles más altos de mortalidad en la infancia se siguen presentando en el interior peninsular, en buena parte de Castilla y Extremadura y en algunas provincias del sur, mientras que en el País Vasco, Cataluña y Baleares el peso de esta mortalidad es menor, frente a una extensa área de provincias que mantienen niveles similares de mortalidad en estas edades.

${ }_{11}$ El coeficiente de variación del ${ }_{5} \mathrm{q}_{0}$ provincial pasa de 18,4 por 100 en 1906 a 21,7 por 100 en 1932 . 


\section{MAPA 1}

Mortalidad $\left({ }_{5} q_{0}\right)$ en España - 1906

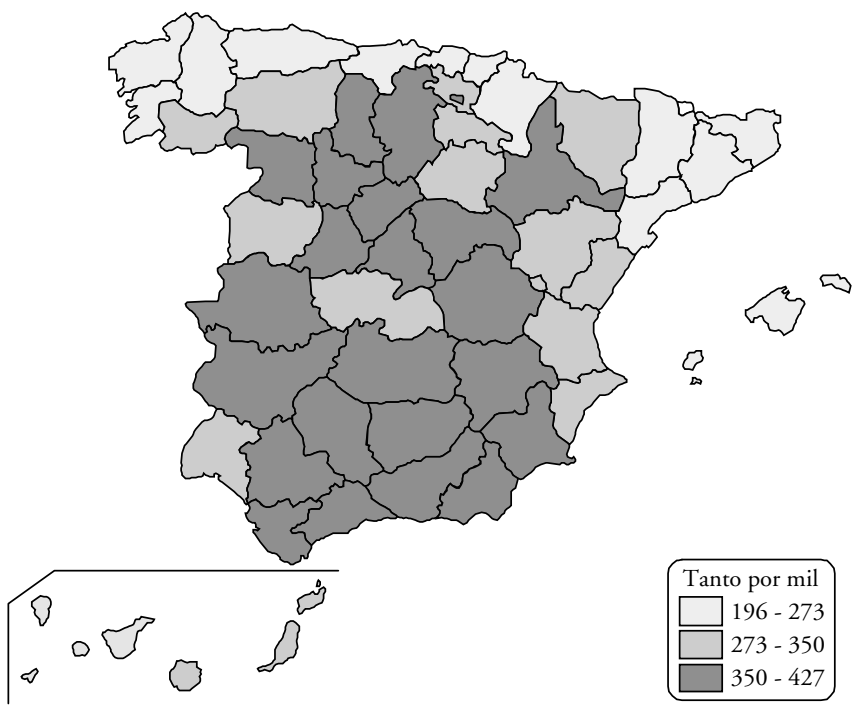

MAPA 2

Mortalidad $\left({ }_{5} q_{0}\right)$ en España - 1932

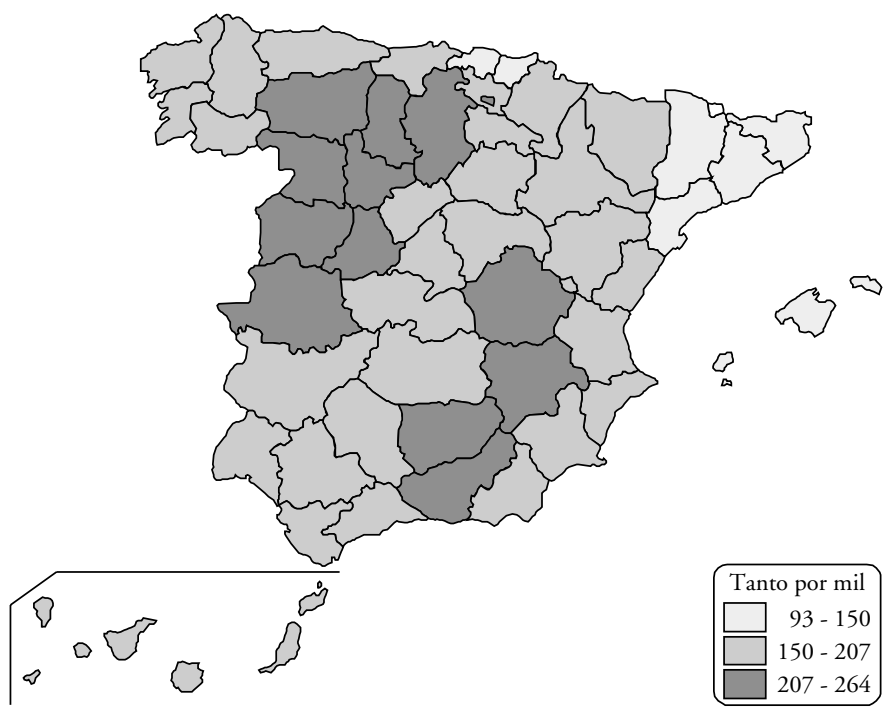


Examinar la relación de estas variaciones geográficas en la mortalidad con la distribución espacial de la mortalidad por causas constituye uno de los planteamientos básicos de este trabajo, y para ello se han elaborado los cocientes provinciales $\left(\mathrm{q}_{0},{ }_{4} \mathrm{q}_{1}, y_{5} \mathrm{q}_{0}\right)$ para cada uno de los grupos de enfermedades utilizados en este trabajo. También se han calculado los porcentajes que los cocientes de los distintos grupos de enfermedades representan dentro del cociente global de mortalidad por edad. Así, por ejemplo, se ha calculado el porcentaje que representan el ${ }_{5} \mathrm{q}_{0}$ por enfermedades del aparato digestivo dentro del ${ }_{5} \mathrm{q}_{0}$ total. De igual manera se ha operado con el resto de grupos de enfermedades y edades. De este modo, tratamos de hacer más visibles los comportamientos regionales de la mortalidad por grupos de causas, apreciándose con este procedimiento una mayor claridad en la distribución espacial de la mortalidad para ciertos tipos de enfermedades. Para nuestro análisis hemos empleado los valores relativos al cociente de mortalidad ${ }_{5} \mathrm{q}_{0}$, que comprende la totalidad de las defunciones ocurridas entre el nacimiento y el quinto aniversario $^{12}$.

Los resultados han permitido observar cierto patrón regional en la distribución de algunas causas de muerte, como las enfermedades del aparato digestivo y las del aparato respiratorio. En cambio, y como ya advirtiera Pascua (1934a: 67-79), en la mortalidad por enfermedades infecto-epidémicas y por enfermedades del sistema nervioso se ha encontrado una gran variación en los niveles de las distintas provincias, siendo imposible apreciar una clara ordenación territorial de las mismas.

Así, en el caso de la mortalidad por enfermedades del aparato digestivo, los resultados (mapas 3 y 4) muestran la distribución provincial según el peso (porcentaje) que este grupo de causas ocupa en la mortalidad ocurrida entre el nacimiento y el quinto cumpleaños.

Ambos mapas no reflejan los niveles, sino la estructura (peso porcentual) de la mortalidad por causas en cada provincia y, en este caso, el peso relativo de las enfermedades digestivas dentro del total de la mortalidad provincial en la infancia. De este modo, no se reflejan los cambios de nivel, sino los cambios en la composición de la mortalidad por causas en cada fecha, siendo posible señalar que en 1932 hay una clara homogeneización en la distribución de esta mortalidad frente a cierta heterogeneidad en $1906^{13}$. En ambas fechas puede apreciarse cómo las defunciones por enfermedades del aparato digestivo mantienen una importancia relativa notable en la mortalidad de buena parte de las provincias del interior y del sur peninsular, sobre todo en las situadas en el margen oeste a lo largo de la frontera con Portugal, exceptuando el caso de Huelva.

${ }_{12}$ La presentación de los resultados para $\mathrm{q}_{0} \mathrm{y}_{4} \mathrm{q}_{1}$ habría dado lugar a una notable proliferación de mapas.

${ }^{13}$ Los valores de los intervalos usados en cada mapa se han establecido de acuerdo a los valores que cada indicador tiene para cada una de las fechas utilizadas, usando la misma amplitud para cada intervalo. 


\section{MAPA 3}

Porcentaje de mortalidad en la infancia $\left({ }_{5} q_{0}\right)$ por enfermedades del aparato digestivo - 1906

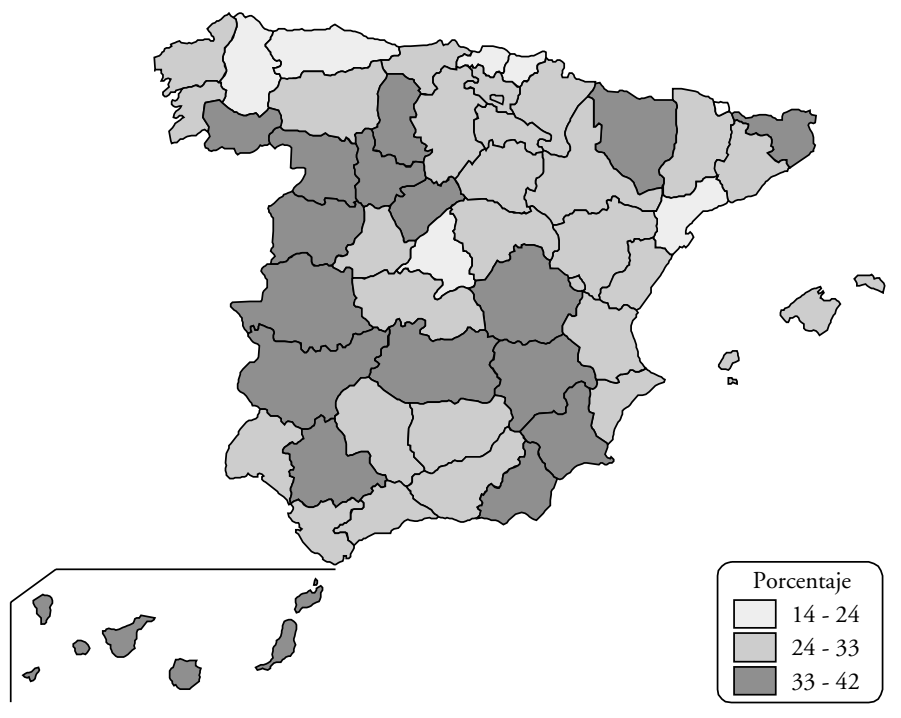

MAPA 4

Porcentaje de mortalidad en la infancia $\left.{ }_{5} q_{0}\right)$ por enfermedades del aparato digestivo - 1932

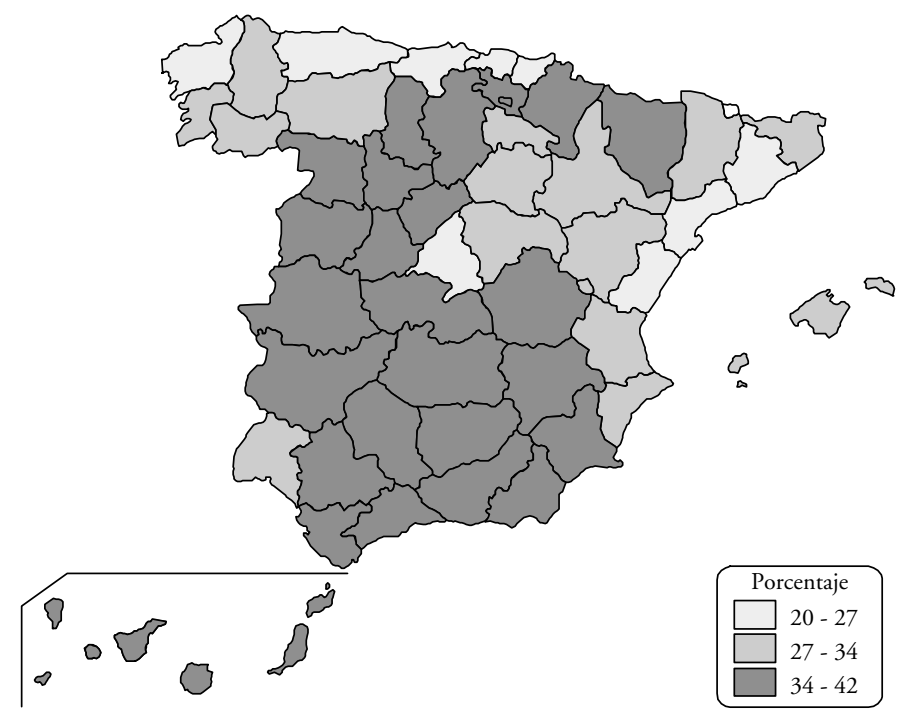


La menor homogeneidad del mapa de enfermedades del aparato digestivo en 1906 se encuentra relacionada con la existencia de una buena proporción de fallecimientos ubicados dentro del grupo de «otras enfermedades» y «enfermedades mal definidas» para este año. También hay que tener en cuenta el hecho de que, en 1906, el patrón epidemiológico de la mortalidad esté fuertemente determinado por otros grupos de enfermedades que experimentan una notable reducción para 1932, como es el caso de las enfermedades infectoepidémicas.

Mención especial hay que hacer de la provincia de Madrid en la mortalidad por enfermedades del aparato digestivo. Como puede apreciarse en los mapas 3 y 4, esta provincia no figura entre las que cuentan con una mayor proporción de casos por estas enfermedades. La explicación radicaría en la menor incidencia que las enfermedades del aparato digestivo suelen tener en aquellos núcleos urbanos donde antes se desarrollan las oportunas medidas higiénicas y sanitarias. En cambio, otras enfermedades, como las del aparato respiratorio, de difícil control en su propagación y contagio, son especialmente importantes en núcleos densamente poblados. Así ocurre en el caso concreto de Madrid, como se verá a continuación.

El peso de las enfermedades del aparato respiratorio dentro de la mortalidad infantil y juvenil (mapas 5 y 6) muestra un patrón distinto y, en buena medida, opuesto al de las enfermedades digestivas. En este caso, la configuración regional en 1906 es más homogénea que en el año 1932. De acuerdo con el mapa 5, el peso relativo de estas enfermedades en la mortalidad infantil y juvenil es mayor en las provincias del norte y este peninsular, destacando los casos especiales de Madrid y Barcelona dentro de esta distribución. La mayor mortalidad urbana por este tipo de enfermedades es la clave de la presencia de ambas provincias, o, más exactamente, sus capitales, en la distribución regional del fenómeno para las dos fechas consideradas. Así, la comparación entre la mortalidad por enfermedades del aparato respiratorio en la capital y el resto provincial revela que, tanto en Madrid como en Barcelona, la mortalidad de la infancia $\left({ }_{5} \mathrm{q}_{0}\right)$ en la capital supera en un 42 por 100 a la del resto de la provincia, situándose ambas capitales entre las 10 primeras ciudades con una mayor mortalidad por infecciones del aparato respiratorio. El tamaño y densidad de la población desempeñarían en este tipo de enfermedades un papel esencial a la hora de entender esta particular distribución regional de la mortalidad.

Si las enfermedades del aparato digestivo y del respiratorio permiten identificar cierto patrón en su distribución espacial, no ocurre lo mismo con las enfermedades infecto-epidémicas y las del sistema nervioso, cuya variabilidad impide apreciar con claridad cualquier perfil geográfico en su incidencia. Por ello, nuestro análisis se centra en el comportamiento de los dos primeros grupos de enfermedades. Así, las diferencias encontradas en la distribución de la mortalidad por enfermedades del aparato digestivo, con mayor peso en el sur y oeste peninsular, frente a la mortalidad por infecciones respiratorias, con mayor presencia en el norte y este del mapa español, hacen pensar en la posibi- 


\section{MAPA 5}

Porcentaje de mortalidad en la infancia $\left({ }_{5} q_{0}\right)$ por enfermedades del aparato respiratorio - 1906

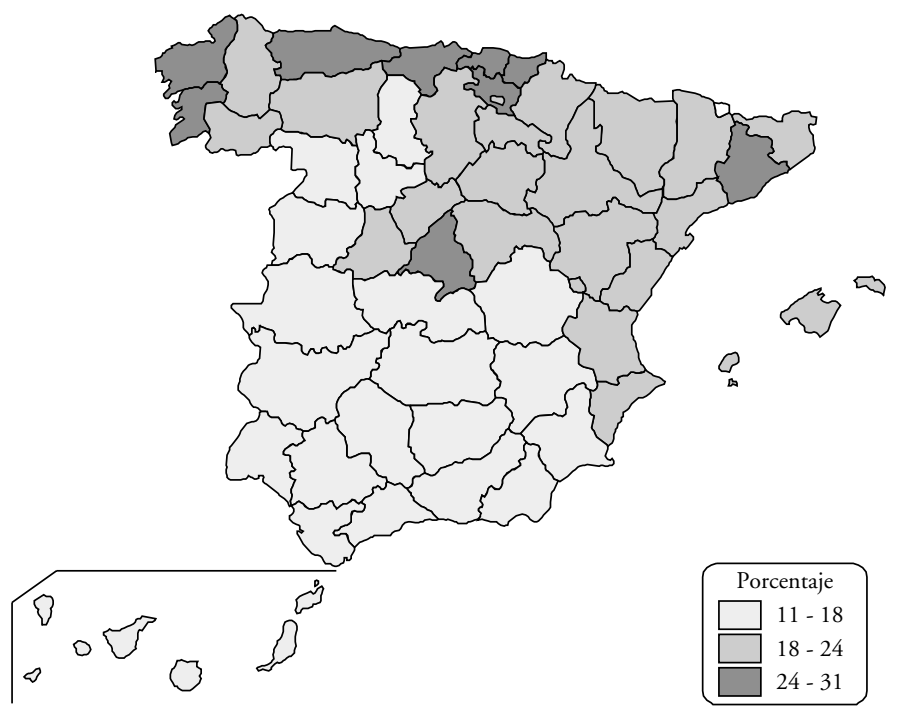

MAPA 6

Porcentaje de mortalidad en la infancia ( $\left.{ }_{5} q_{0}\right)$ por enfermedades del aparato respiratorio - 1932

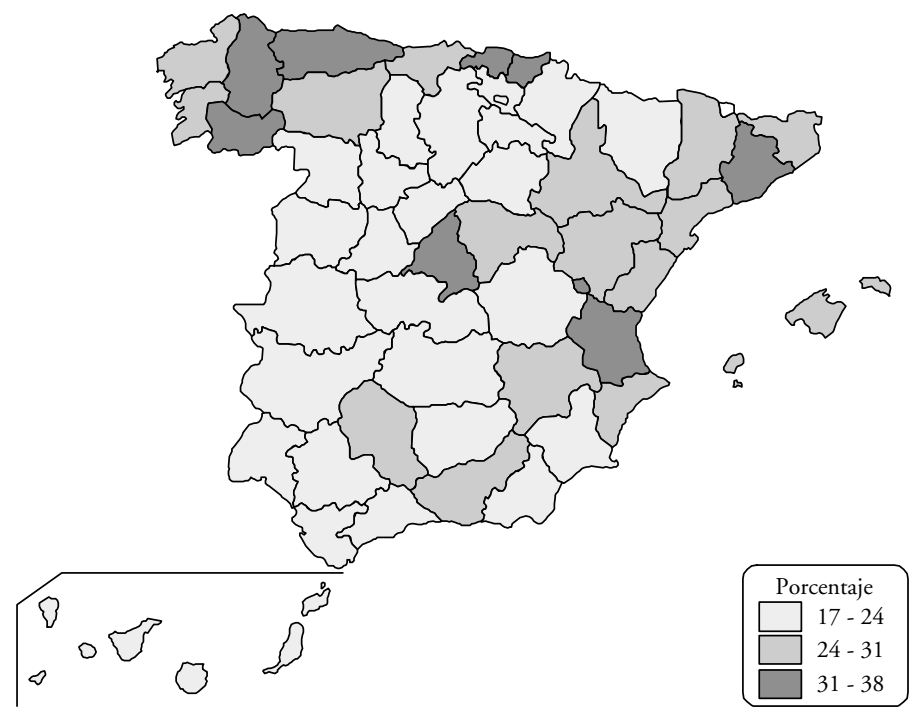


lidad de cierta relación con distintas variables, entre las que cabría destacar las variaciones climáticas de unas a otras provincias.

En 1962 Antonio Arbelo incluyó en su estudio un apartado dedicado a la relación entre la mortalidad infantil y el clima, aunque se centró principalmente en los datos disponibles para el archipiélago canario. Recientes trabajos han señalado la relación existente entre la estructura de la mortalidad infantil y juvenil y las diferencias en el patrón de lluvias y temperaturas en España (Ramiro Fariñas y Sanz Gimeno, 2000a: 75-78; 2000b: 245-249). Naturalmente, la correspondencia entre estas variables climáticas y la distribución de la mortalidad por causas de muerte viene mediatizada por diversos factores como son la calidad del agua y la naturaleza y comportamiento de los microorganismos infecciosos. Incluso cabría hablar de interrelaciones mucho más complejas entre determinantes sociales y culturales en los que la variable climática no siempre tiene un papel fácil de definir. De hecho, la asociación estadística entre mortalidad y clima no ofrece siempre resultados lo suficientemente evidentes. En el análisis realizado para este trabajo se han obtenido algunos indicios que confirman la existencia de cierto grado de asociación entre la distribución provincial de la mortalidad por causas y las diferencias en las temperaturas provinciales ${ }^{14}$. Éste es el caso de la correlación realizada entre temperaturas veraniegas y enfermedades del aparato respiratorio en 1906, que arroja un coeficiente de $-0,483$ (nivel de significación 0,01 ), indicando la relación existente entre una menor temperatura veraniega y un mayor peso de las enfermedades respiratorias en el mapa provincial.

Pese a la importancia de la evidencia climática, hay que tener en cuenta el papel que otros factores sociales, culturales y económicos juegan en la distribución regional de la mortalidad por causas de muerte. La relación con otros aspectos de la modernización social y económica también tiene importantes implicaciones, como en el caso de los procesos de industrialización de algunas provincias y ciudades o la mejora de las tasas de alfabetización femenina y la divulgación de los preceptos médicos e higiénicos.

Algunas evidencias sobre la existencia de una vinculación entre modernización y mejoras higiénico-sanitarias se pueden apreciar al examinar la distribución provincial del descenso de la mortalidad (mapa 7) con la misma distribución del descenso de la mortalidad por causas del aparato digestivo (mapa 8). Ambos mapas muestran el porcentaje provincial de reducción de la mortalidad en la infancia $\left({ }_{5} q_{0}\right)$ entre 1906 y 1932. Como puede observarse, existe una gran asociación en la caída de la mortalidad infantil y juvenil y la producida en la mortalidad por enfermedades del aparato digestivo en las mismas edades y período. Los mayores descensos de la mortalidad se producen en algunas de las provincias y regiones que venían experimentando cierto adelanto en su proceso

${ }_{14}$ Ante las dificultades para tener una muestra completa a nivel provincial de temperaturas medias en 1906 y 1932, se han utilizado los datos que el Instituto Nacional de Meteorología (s.f.) ofrece para el período de 1961 a 1990 . Se ha supuesto que las variaciones geográficas de las temperaturas no se han modificado sustancialmente a lo largo del siglo XX. 


\section{MAPA 7}

Porcentaje de reducción de la mortalidad en la infancia $\left({ }_{5} q_{0}\right)$ de 1906 a 1932

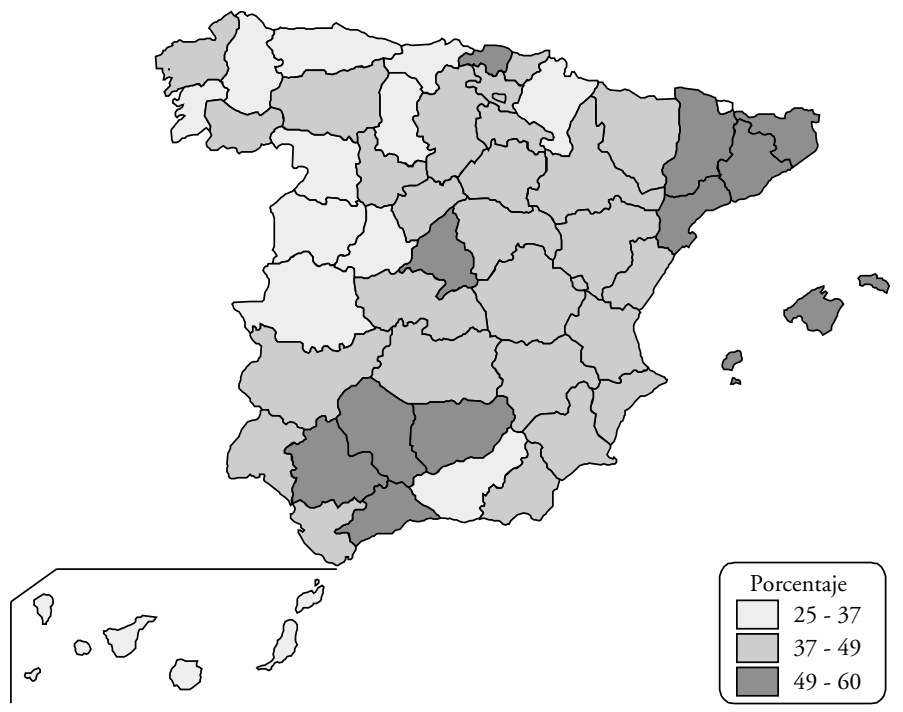

MAPA 8

Porcentaje de reducción de la mortalidad $\left({ }_{5} q_{0}\right)$ por enfermedades del aparato digestivo de 1906 a 1932

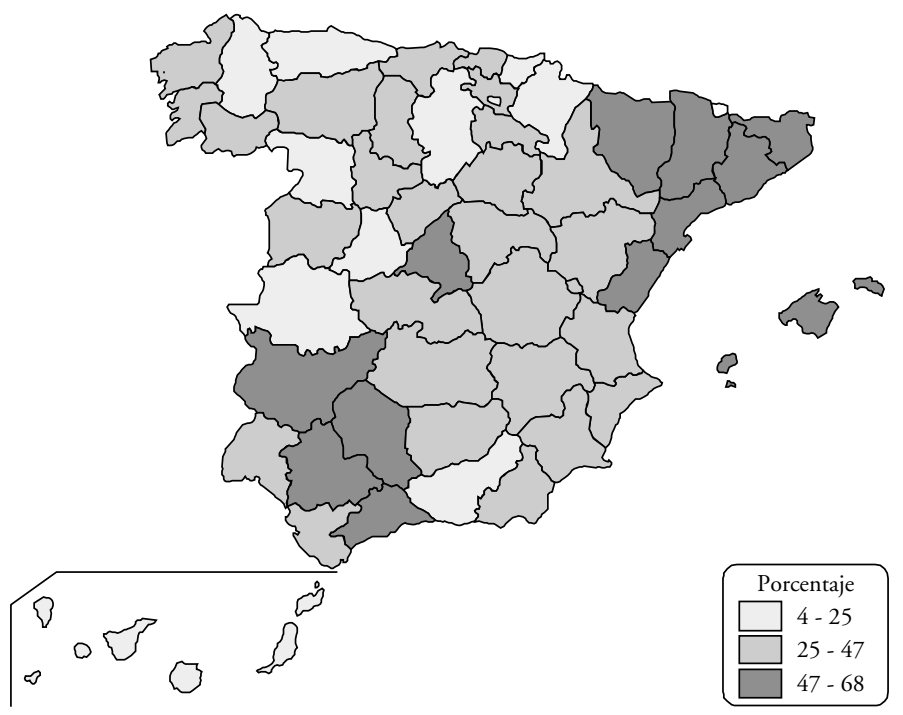


de modernización y mejora de las condiciones higiénico-sanitarias, tales como Madrid, Vizcaya, Cataluña y Baleares. Sin embargo, también hay reducciones importantes de la mortalidad en algunas provincias andaluzas como Córdoba, Jaén, Málaga y Sevilla, que no se encuentran en la misma situación de desarrollo que los casos antes citados. Tales resultados no invalidan, en absoluto, la asociación entre modernización y caída de la mortalidad, pero sí introducen claramente la necesidad de incorporar otros elementos explicativos al discurso y de ahondar, aún más, sobre las características que el proceso de caída de la mortalidad ha tenido en las distintas regiones españolas.

Esta estrecha relación entre la distribución provincial del descenso de la mortalidad en el primer tercio del siglo Xx y la caída de la mortalidad en enfermedades del aparato digestivo destaca la necesidad de investigar más de cerca las diferencias urbano-rurales del fenómeno y, también, analizar con detalle el papel de las pautas sociales, culturales y demográficas ligadas al proceso de modernización. Este tipo de análisis contribuiría a explicar la relación entre este fenómeno y el desarrollo de medidas higiénico-sanitarias en ciudades como Madrid y Barcelona o en la misma área de Cataluña, que presentan, como ya se ha indicado, un claro avance en el proceso de transición y descenso de la mortalidad. También nos permitiría explicar las razones de una importante caída de la mortalidad en algunas zonas del mapa de España que no se caracterizan por su papel pionero en este proceso de modernización y desarrollo.

\section{CONCLUSIONES}

El fuerte peso de enfermedades como las del aparato digestivo y las del aparato respiratorio, unido a la presión que ejercían las enfermedades infectoepidémicas, se han destacado como factores esenciales para entender la peculiar estructura de la mortalidad en la infancia en los inicios de la transición, cuando la mortalidad juvenil $\left({ }_{4} \mathrm{q}_{1}\right)$ se sitúa en niveles similares a la infantil $\left(\mathrm{q}_{0}\right)$. La notable reducción de las enfermedades infecto-epidémicas y la caída de las afecciones del aparato digestivo explican la mayor parte del intenso descenso experimentado en las defunciones durante la infancia. La disminución del peso de enfermedades como la diarrea y enteritis, así como la erradicación de la viruela y la difteria y la importante reducción de la mortalidad por sarampión, aparecen como elementos fundamentales para entender el cambio que se produjo en la estructura de la mortalidad en los primeros años de vida.

Los cambios en el patrón epidemiológico también expresan importantes transformaciones sociales y económicas ${ }^{15}$ en la sociedad española de principios del siglo XX. Mientras que el papel de la intervención médico-terapéutica se reduce prácticamente a casos como el de la viruela y, en menor grado, la difteria, la notable reducción de la mortalidad por enfermedades digestivas pone de

15 En torno al papel desempeñado por los cambios económicos en la caída de la mortalidad, véase el trabajo de Reher y Sanz (2000). 
manifiesto la importancia que otros elementos, ligados a un cambio en la valoración social del niño y a los avances en salud pública, han tenido a la hora de explicar el declive de la mortalidad. Los resultados han señalado la importancia de las mejoras higiénico-sanitarias en el ámbito urbano, donde se inician con mayor antelación y rapidez los cambios que favorecen la reducción de la mortalidad. Este hecho, junto a otras evidencias aportadas por este y otros trabajos realizados sobre el tema, permiten atribuir un papel destacado a la transformación de las actitudes sociales acerca del problema que supone la mortalidad de la infancia en esta etapa del proceso de desarrollo y modernización. Además, conviene tener en cuenta cambios en aspectos tan esenciales como la atención y cuidado dispensados por la madre que, sin duda, están directamente implicados en la importante disminución que presentan tanto las enfermedades digestivas como las del aparato respiratorio y las infecto-epidémicas.

El declive de la mortalidad en la infancia, y especialmente la mayor reducción de la mortalidad en los niños de 1 a 4 años, se explica, en buena medida, por la mejora en aspectos tales como la alimentación y unos cuidados más adecuados frente a problemas como la dentición o frente a procesos morbosos que, en un contexto de menor desarrollo, hubieran supuesto una elevada probabilidad de muerte, como en el caso del sarampión.

El análisis de la distribución provincial de la mortalidad ha contribuido también a destacar la importancia de estos elementos en la evolución de la mortalidad durante la infancia, sobre todo en lo que respecta al patrón regional de las enfermedades del aparato digestivo y también, aunque en menor medida, de las enfermedades del aparato respiratorio. Frente a una zona norte y este donde se da un peso importante de la mortalidad por causas respiratorias, destacan una zona sur y oeste en la que las enfermedades del aparato digestivo ejercen una notable influencia. Asimismo, se ha encontrado una estrecha similitud en la distribución provincial del descenso de la mortalidad general y la caída de las enfermedades del aparato digestivo, dándose algunas evidencias de la importancia del crecimiento económico y el desarrollo social como claves del éxito en la lucha contra la mortalidad de la infancia en unas regiones del país frente a otras. Sin embargo, también se han encontrado casos que, sin invalidar la relevancia del proceso de modernización social y económica, precisan de la incorporación de otras variables explicativas y de otros estudios en profundidad acerca de las peculiaridades regionales del proceso de declive de la mortalidad.

Pese a la relevancia de estos resultados, quedan aún un buen número de cuestiones por tratar, que precisan de análisis más exhaustivos, no sólo de las diferencias geográficas, sino también de las disparidades existentes entre el mundo urbano y rural y su relación con los factores que intervienen en el descenso de la mortalidad. Determinar claramente el papel de factores como el clima, la educación de la mujer, el desarrollo de la sanidad pública o la propia estructura del sistema productivo en la evolución de la mortalidad infantil y juvenil, teniendo en cuenta las diferencias regionales del fenómeno, son, sin duda, algunas de las asignaturas pendientes. 


\section{REFERENCIAS BIBLIOGRÁFICAS}

Arbelo Curbelo, A. (1962), La mortalidad de la infancia en España, 1901-1950, Madrid: Consejo Superior de Investigaciones Científicas, Dirección General de Sanidad.

Barona, J. L., y BAREA, E. (1996), "Enfermedad y muerte en la ciudad de Valencia (19011920)», Boletín de la Asociación de Demografía Histórica, XIV, II: 57-82.

Bernabeu Mestre, J. (1994), "Problèmes de santé et causes de décès infantiles en Espagne (1900-1935)", Annales de Démographie Historique, 61-77.

Bertillon, J. (1899), Nomenclatura de las enfermedades (causas de las defunciones por...), Madrid: Dirección General del Instituto Geográfico y Estadístico.

Burnet, M., y White, D. O. (1982), Historia natural de la enfermedad infecciosa, Madrid: Alianza Editorial.

Dopico Gutiérrez Del Arroyo, F. (1985), «Desarrollo económico y social y mortalidad infantil. Diferencias regionales (1900-1950)», Crisis, Autonomías y Desarrollo Regional, I: 357-372.

Dopico, F., y ReHer, D. S. (1998), El declive de la mortalidad en España, 1860-1930, Monografía 1, Asociación de Demografía Histórica.

ECheVerri Dávila, B. (1996), La gripe española. La pandemia de 1918-1919, Madrid: Centro de Investigaciones Sociológicas, Siglo XXI.

GÓmez Redondo, R. (1992), La mortalidad infantil española en el siglo XX, Madrid: Centro de Investigaciones Sociológicas, Siglo XXI.

Hauser, P. (1902), Madrid bajo el punto de vista médico-social. Su morbicidad y mortalidad en relación con las enfermedades infecciosas y su profilaxis, con el estado meteorológico, con las condiciones higiénicas y con las económico-sociales, su epidemiología en relación con la policía sanitaria, Madrid: Edición de Carmen García del Moral (1979), Editora Nacional.

Instituto Nacional de Meteorología (s.f.), Guía resumida del clima en España, 1961-1990, Madrid: Instituto Nacional de Meteorología, Ministerio de Medio Ambiente.

Livi-BaCCI, M. (1987), Ensayo sobre la historia demográfica europea. Población y alimentación en Europa, Barcelona: Ariel.

MCKeown, T. (1978), El crecimiento moderno de la población, Barcelona: Antoni Bosch.

NADAl Oller, J. (1991), La población española (siglos XVI al XX), Barcelona: Ariel.

Notestein, Frank W. (1945), «Population: the long view», en T. W. Schultz (ed.), Food for the world, Chicago: Chicago University Press, pp. 36-57.

Omran, A. R. (1971), "The epidemiologic transition. A theory of the epidemiology of population change», Milbank Men. Fund. Quaterly, 49: 509-538.

PASCUA, M. (1934a), La mortalidad infantil en España, Madrid: Departamento de Estadísticas Sanitarias de la Dirección General de Sanidad.

- (1934b), Mortalidad específica en España, Madrid: Dirección General de Sanidad.

Perdiguero Gil, E. (1993), "Causas de muerte y relación entre conocimiento científico y conocimiento popular", Boletín de la Asociación de Demografía Histórica, XI, 3, 65-88.

PÉrez MoredA, V. (1985), "La evolución demográfica española en el siglo XIX (1797-1930): tendencias generales y contrastes regionales», en La popolazione italiana nell'ottocento, Bolonia: CLUEB, SIDES, pp. 45-114.

RAMIRo FARIÑAS, D. (1998), La evolución de la mortalidad en la infancia en la España interior, 1785-1960, Madrid: Tesis doctoral inédita, Universidad Complutense de Madrid.

Ramiro Fariñas, D., y Sanz Gimeno, A. (1999), "Cambios estructurales en la mortalidad infantil y juvenil española, 1860-1990», Boletín de la Asociación de Demografía Histórica, XVII, I: 49-87.

Ramiro Fariñas, D., y SAnZ Gimeno, A. (2000a), «Structural changes in childhood mortality in Spain, 1860-1990", International Journal of Population Geography, 6, 1: 61-82.

- (2000b), "Childhood mortality in Central Spain, 1790-1960. Changes in the course of demographic modernisation", Continuity and Change, 15, 2: 235-267.

ReHer, D. (1996), La familia en España. Pasado y presente, Madrid: Alianza Universidad. 
Reher, D., y Valero Lobo, A. (1995), Fuentes de información demográfica en España, Madrid: Centro de Investigaciones Sociológicas.

Reher, D. S.; Pérez Moreda, V., y Bernabeu Mestre, J. (1997), «Assessing change in historical context. Childhood mortality patterns in Spain during the demographic transition», en C. A. Corsini y P. P. Viazzo (eds.), The decline of infant and child mortality: The European experience: 1750-1990, La Haya: Martinus Nijhoff Publishers, pp. 35-56.

Reher, D. S., y SANZ Gimeno, A. (2000), «Mortality and economic development over the course of modernization: An analysis of short-run fluctuations in Spain, 1850-1990", Population Studies, 54, 2: 135-152.

Revenga, R. (1904), La muerte en España. Estudio estadistico sobre la mortalidad, Madrid: Sociedad Española de Higiene, Imprenta de la Prensa de Madrid.

Robles GonzÁlez, E.; Bernabeu Mestre, J., y Benavides, F. G. (1996), «La transición sanitaria. Una revisión conceptual», Boletín de la Asociación de Demografía Histórica, XIV, 1: 117 144.

Rodríguez OcaÑa, E. (1996), "Una medicina para la infancia», en J. M. Borrás Llop (dir.), Historia de la infancia en la España contemporánea, 1834-1936, Madrid: Ministerio de Trabajo y Asuntos Sociales, Fundación Germán Sánchez Ruipérez, pp. 149-179.

Sanz Gimeno, A. (1999), La mortalidad de la infancia en Madrid. Cambios demográfico-sanitarios en los siglos XIX y XX, Madrid: Consejería de Sanidad y Servicios Sociales, Comunidad de Madrid.

Sanz Gimeno, A., y Ramiro Fariñas, D. (2000), "The decline of childhood mortality in Central Spain by causes of death, 1860-1960», en European Social Science History Conference, Amsterdam, 12-15 abril.

Ulecia CARDONA, R. (1903), «Mortalidad en la primera infancia», en E. Rodríguez Ocaña (ed.) (1987), La constitución de la medicina social como disciplina en España (1882-1923), Madrid: Ministerio de Sanidad y Consumo, pp. 161-176.

Vallin, J. (1987), Seminario sobre causas de muerte. Aplicación al caso de Francia, Santiago de Chile: Centro Latinoamericano de Demografía (CELADE).

\begin{abstract}
This article is a contribution to the study of the decline of childhood mortality (ages 0 to 4 ) in Spain during the beginning of the xx century. The work analyses the mortality levels and structure by age $\left(\mathrm{q}_{0}\right.$ and $\left.{ }_{4} \mathrm{q}_{1}\right)$ and cause of death in two key dates: 1906 and 1932, according to the main groups of causes (digestive diseases, respiratory diseases, infectious and epidemic diseases and nervous system diseases). The work reveals the importance of this analysis to explain the changes in mortality by age and cause of death during the beginning of the demographic transition and the social and economic modernization of the country. It also examines the regional distribution of the fenomenon and shows the extent of regional differences in demographic modernisation.
\end{abstract}

